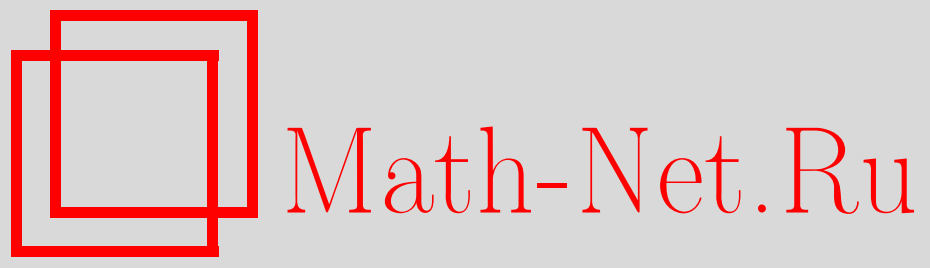

V. I. Paulauskas, D. Surgailis, On the rate of approximation in limit theorems for sums of moving averages, Теория вероятн. и ее примен., 2007, том 52, выпуск 2, 405-414

DOI: https://doi.org/10.4213/tvp185

Использование Общероссийского математического портала Math-Net.Ru подразумевает, что вы прочитали и согласны с пользовательским соглашением

http://www . mathnet.ru/rus/agreement

Параметры загрузки:

IP : 54.198 .67 .100

26 апреля 2023 г., 13:17:03 
4. Данфорд Н., Швари Дж. Линейные операторы: Общая теория. М.: ИЛ, 1962, $895 \mathrm{c}$.

5. Гладышев Е.Г. О периодически коррелированных случайных последовательностях. - Докл. АН СССР, 1961, т. 137, № 5, с. 1026-1029.

6. Makagon A., Miamee A. G., Salehi H. Periodically correlated processes and their spectrum. - Nonstationary Stochastic Processes and Their Applications. River Edge: World Scientific, 1992, p. 147-164.

7. Makagon A., Salehi $H$. Is the spectral domain of a periodically correlated sequence complete? Technical Report RM-529. Department of Statistics and Probability, Michigan State University, 1993.

8. Miamee A. G. Periodically correlated processes and their stationary dilations. - SIAM J. Appl. Math., 1990, v. 50, № 4, p. 1194-1199.

9. Miamee A. G., Salehi $H$. An example of a harmonizable process whose spectral domain is not complete. - Scand. J. Statist., 1991, v. 18, № 3, p. 249-254.

10. Miamee A.G., Soltani A.R. On spectral analysis of periodically correlated stochastic processes. Technical Report. Department of Mathematics, Hampton University, 1995.

11. Rao M. M. The spectral domain of multivariate harmonizable processes. - Proc. Natl. Acad. Sci. USA, 1984, v. 81, p. 4611-4612.

12. Розанов Ю.А. Стационарные случайные процессы. М.: Физматгиз, 1963, 284 с.

13. Soltani A.R. A characterization theorem for stable random measures. - Stochastic Anal. Appl., 2000, v. 18, № 3, p. 299-308.

14. Soltani A.R., Shishebor $Z$. A spectral representation for weakly periodic sequences of bounded linear transformations. - Acta Math. Hungar., 1998, v. 80, № 3, p. 265-270.

15. Wiener N., Masani P. The prediction theory of multivariate stochastic processes. II: The linear predictor. - Acta Math., 1958, v. 99, p. 93-137.

Поступила в редакцию

9.III. 2002

Исправленный вариант

29.VII.2004

(C) $2007 \mathrm{r}$.

PAULAUSKAS V.*, SURGAILIS D.*

\section{ON THE RATE OF APPROXIMATION IN LIMIT THEOREMS FOR SUMS OF MOVING AVERAGES ${ }^{1)}$}

Рассматривается линейный процесс $X_{t}=\sum_{j=0}^{\infty} a_{j} \varepsilon_{t-j}, t \geqslant 1$, где $\varepsilon_{i}$, $i \in \mathbf{Z}$, - независимые одинаково распределенные случайные величины, принадлежащие области притяжения устойчивого закона с индексом $\alpha$, $0<\alpha \leqslant 2, \alpha \neq 1$. Накладывая условия на величины $\varepsilon_{i}$ и коэффициенты $a_{j}$, мы оцениваем точность аппроксимации распределений сумм $S_{n}=$ $B_{n}^{-1} \sum_{t=1}^{n} X_{t}$ соответствующими устойчивыми законами. Полученные оценки имеют правильный порядок относительно $n$.

Ключевые слова и фразы: тинейные процессы, устойчивые законы, точность аппроксимации.

* Vilnius University, Department of Mathematics and Informatics, Naugarduko, 24, Vilnius 03225, Lithuania; Institute of Mathematics and Informatics, Akademijos, 4, Vilnius, Lithuania; e-mail: vygantas.paulauskas@maf.vu.lt; sdonatas@ktl.mii.lt

1) The research was supported by the bilateral France-Lithuania scientific project Gilibert and the Lithuanian State Science and Studies Foundation, grant № T-10/06. 
1. Introduction. Consider a moving average process

$$
X_{t}=\sum_{j=0}^{\infty} a_{j} \varepsilon_{t-j}
$$

where $\left(\varepsilon_{i}, i \in \mathbf{Z}\right)$ are independent and identically distributed (i.i.d.) random variables such that $X_{t}$ is well defined, that is, the series in (1) converges a.s., or equivalently, in probability. Linear processes as in (1) form a natural class of stationary time series models and include popular parametric classes such as ARMA and ARFIMA. Depending on the rate of decay of the coefficients $a_{j}$, the stationary variables $X_{t}$ in (1) can be weakly or strongly dependent. Usually, weak dependence refers to absolutely convergent coefficients $\left(\sum_{j=0}^{\infty}\left|a_{j}\right|<\infty\right)$ and strong (or long-range) dependence to the absolutely divergent series $\left(\sum_{j=0}^{\infty}\left|a_{j}\right|=\infty\right)$. The fundamental Wold decomposition says that every regular stationary Gaussian process can be represented in the form (1), with i.i.d. standard normal innovations $\varepsilon_{i}, i \in \mathbf{Z}$.

Let

$$
S_{n}:=B_{n}^{-1} \sum_{t=1}^{n} X_{t}, \quad S_{n}(\tau):=B_{n}^{-1} \sum_{t=1}^{[n \tau]} X_{t} \quad(\tau \in[0,1]),
$$

where $B_{n}$ is a normalization. Limit behavior of sums $S_{n}$ and partial sums $S_{n}(\tau), \tau \in[0,1]$, of linear processes in (1) is well investigated. Several authors (see [5], [7], [16]) discussed the (functional) convergence of the partial sums process in linear variables $X_{t}$ with finite variance, to a fractional Brownian motion $B_{H}(\tau)$ with index $0<H<1$. Convergence of partial sums of moving averages of i.i.d. r.v.'s with infinite variance to an $\alpha$-stable $(0<\alpha<2)$ fractional motion was studied in [2], [11], [3] and other papers. Let us note that convergence of the partial sums process $S_{n}(\tau)$ to a self-similar process (e.g., a fractional Brownian motion) requires a regular growth of normalizing constants $B_{n}$, see [10]. On the other hand, a central limit theorem for (simple) sums $S_{n}$ of linear variables as in (1) holds under general assumptions $\mathbf{E} \varepsilon_{0}^{2}<\infty, \mathbf{E} \varepsilon_{0}=0$, and $B_{n}^{2}=\mathbf{E}\left(\sum_{t=1}^{n} X_{t}\right)^{2} \rightarrow \infty$; see [8], also [9, Theorem 18.2].

Let $\left(\varepsilon_{i}\right)$ in (1) belong to the domain of attraction of $\alpha$-stable r.v. $\eta, 0<\alpha \leqslant 2$. Then it is natural to approximate $S_{n}$ and $S_{n}(\tau)$ in (2) by $\alpha$-stable sums

$$
Z_{n}:=B_{n}^{-1} \sum_{t=1}^{n} Y_{t}, \quad Z_{n}(\tau):=B_{n}^{-1} \sum_{t=1}^{[n \tau]} Y_{t} \quad(\tau \in[0,1])
$$

respectively, where

$$
Y_{t}:=\sum_{j=0}^{\infty} a_{j} \eta_{t-j}
$$

and $\left(\eta_{i}, i \in \mathbf{Z}\right)$ are i.i.d. copies of $\alpha$-stable r.v. $\eta$. In many cases, e.g., if $\eta$ is symmetric or the moving average coefficients $\left(a_{j}\right)$ in (1) are nonnegative, the normalization $B_{n}$ in (1)-(4) can be chosen so that the distribution of $Z_{n}$ does not depend on $n$ and coincides with the limit distribution of $S_{n}$.

The aim of this note is to obtain a uniform rate of $\alpha$-stable approximation of $S_{n}$, namely, the decay rate as $n \rightarrow \infty$ of the quantity

$$
\Delta_{n}:=\sup _{x \in \mathbf{R}}\left|\mathbf{P}\left\{S_{n} \leqslant x\right\}-\mathbf{P}\left\{Z_{n} \leqslant x\right\}\right| .
$$

Convergence rates in a functional central limit theorem (with the limiting fractional Brownian motion) were obtained in [7] and [1] (the latter paper contains also some other unpublished references). The reasons for our studying the relatively simple «onedimensional» quantity $\Delta_{n}$ in (5) are the following. Firstly, as mentioned above, a limit distribution of (simple) sums $S_{n}$ exists under much less restrictive conditions on coefficients $\left(a_{j}\right)$ as compared to partial sums $S_{n}(\tau)$; moreover, our results apply to situations when $\mathbf{P}\left\{S_{n} \leqslant x\right\}$ and $\mathbf{P}\left\{Z_{n} \leqslant x\right\}$ do not converge; see Example 2. Secondly, the results in [7] and [1], obtained under the usual assumption $\mathbf{E}\left|\varepsilon_{0}\right|^{3}<\infty$ and some regularity assumptions on $\left(a_{j}\right)$, give a functional convergence rate not better than $n^{-1 / 8}$, while for the quantity $\Delta_{n}$ one can expect under similar assumptions a much better convergence 
rate $n^{-1 / 2}$, similarly as in the i.i.d. case. Indeed, it turns out that in many cases the rate of convergence of $\Delta_{n}$ is the same as in the case of i.i.d. summands, namely $\Delta_{n}=O\left(n^{-\delta / \alpha}\right)$, assuming the existence of $(2+\delta)$-(pseudo)moment of $\varepsilon_{0}, 0<\delta \leqslant 1$ (see Theorems 1-3 and Assumption $\mathrm{D}(\alpha, \delta)$ below for precise formulations). The above mentioned fact is not surprising, since $S_{n}$ can be represented as a weighted sum of i.i.d. r.v.'s $\varepsilon_{i}, i \leqslant n$. We also obtain easily verifiable conditions on the coefficients $\left(a_{j}\right)$ in various dependence situations (the so-called cases of short, long or negative memory of $\left(X_{t}\right)$ ) under which the above mentioned rate of convergence is achieved.

2. Rate of convergence in the central limit theorem for weighted sums of i.i.d. r.v.'s. Let $0<\alpha \leqslant 2, \alpha \neq 1$, and let $\varepsilon$ be a r.v. Write $\varepsilon \in \mathrm{D}(2)(\alpha=2)$ if $\mathbf{E} \varepsilon=0$, $\sigma^{2}:=\mathbf{E} \varepsilon^{2}<\infty$, and $\varepsilon \in \mathrm{D}(\alpha)(0<\alpha<2)$ if there exist constants $c_{\varepsilon}^{ \pm} \geqslant 0, c_{\varepsilon}^{+}+c_{\varepsilon}^{-}>0$, such that

$$
\mathbf{P}\{\varepsilon>x\} \sim c_{\varepsilon}^{+} x^{-\alpha} \quad(x \rightarrow \infty), \quad \mathbf{P}\{\varepsilon<x\} \sim c_{\varepsilon}^{-}|x|^{-\alpha} \quad(x \rightarrow-\infty) ;
$$

moreover, $\mathbf{E} \varepsilon=0$ for $1<\alpha<2$. Condition $\varepsilon \in \mathrm{D}(\alpha)$ means that r.v. $\varepsilon$ belongs to the domain of normal attraction of $\alpha$-stable distribution (see [9]); in other words, if $\varepsilon_{1}, \varepsilon_{2}, \ldots$ are independent copies of r.v. $\varepsilon$, then

$$
n^{-1 / \alpha} \sum_{i=1}^{n} \varepsilon_{i} \stackrel{\text { law }}{\longrightarrow} \eta
$$

where $\eta$ is $\alpha$-stable r.v. with the characteristic function $\mathbf{E e}^{\mathrm{i} u \eta}=\exp \left\{-|u|^{\alpha} \omega(u)\right\}$. Here

$$
\omega(u):= \begin{cases}\frac{\sigma^{2}}{2}, & \alpha=2, \\ \frac{\Gamma(2-\alpha)}{1-\alpha}\left(\left(c_{\varepsilon}^{+}+c_{\varepsilon}^{-}\right) \cos \frac{\pi \alpha}{2}-i\left(c_{\varepsilon}^{+}-c_{\varepsilon}^{-}\right) \operatorname{sign}(u) \sin \frac{\pi \alpha}{2}\right), & \\ 0<\alpha<2, \quad \alpha \neq 1 .\end{cases}
$$

We exclude the case $\alpha=1$ from consideration simply for technical reasons, since in the case $\alpha=1$ centering and normalization are different from those for the rest of values of $\alpha$.

In order to obtain a rate of convergence in the central limit theorem for sums and weighted sums of i.i.d. r.v.'s in $\alpha$-stable domain of attraction, further conditions on the distribution of $\varepsilon$ must be imposed. Recall that pseudomoment of order $\alpha+\delta>\alpha$ of r.v. $\varepsilon \in \mathrm{D}(\alpha)$ is defined by

$$
\kappa_{\alpha, \delta}(\varepsilon):=\int_{\mathbf{R}}|x|^{\alpha+\delta}|\mathrm{d}(\mathbf{P}\{\varepsilon \leqslant x\}-\mathbf{P}\{\eta \leqslant x\})|,
$$

where $\eta$ is the $\alpha$-stable r.v. in (7). For $\varepsilon \in \mathrm{D}(\alpha), 0<\alpha \leqslant 2, \alpha \neq 1,0<\delta \leqslant 1$, introduce the following assumption.

A s s u mption $\mathrm{D}(\alpha, \delta)$. (i) If $\alpha=2$, then $\varepsilon \in \mathrm{D}(2)$ and $\mu_{2+\delta}:=\mathbf{E}|\varepsilon|^{2+\delta}<\infty$.

(ii) If $0<\alpha<1$, then $\varepsilon \in \mathrm{D}(\alpha)$ and $\kappa_{\alpha, \delta}(\varepsilon)<\infty$. Moreover, if $\alpha+\delta>1$, then

$$
\int_{\mathbf{R}} x \mathrm{~d}(\mathbf{P}\{\varepsilon \leqslant x\}-\mathbf{P}\{\eta \leqslant x\})=0 .
$$

(iii) If $1<\alpha<2$, then $\varepsilon \in \mathrm{D}(\alpha), \mathbf{E} \varepsilon=0$, and $\kappa_{\alpha, \delta}(\varepsilon)<\infty$. Moreover, if $\alpha+\delta>2$, then

$$
\int_{\mathbf{R}} x^{2} \mathrm{~d}(\mathbf{P}\{\varepsilon \leqslant x\}-\mathbf{P}\{\eta \leqslant x\})=0 .
$$

Consider weighted sums

$$
S_{n}=B_{n}^{-1} \sum_{i \leqslant n} b_{n, i} \varepsilon_{i}, \quad Z_{n}=B_{n}^{-1} \sum_{i \leqslant n} b_{n, i} \eta_{i}
$$

where $\varepsilon_{i}, i \leqslant n$, are i.i.d. copies of $\varepsilon$ and $\eta_{i}, i \leqslant n$, are i.i.d. copies of $\eta$, coefficients $b_{n, i}$, $i \leqslant n$, are real, and normalizing sequence is defined by

$$
B_{n} \equiv B_{n}(\alpha):=\left(\sum_{i \leqslant n}\left|b_{n, i}\right|^{\alpha}\right)^{1 / \alpha} \text {. }
$$


Here and in what follows $\sum_{i \leqslant n}=\sum_{i=-\infty}^{n}$. Without loss of generality we assume that $\sigma^{2}=1$ in the case $\alpha=2$. Note that $Z_{n}$ has $\alpha$-stable distribution, which does not depend on $n$ (and coincides with the distribution of $\eta$ ) if either $\eta$ is symmetric, or weights $b_{n, i}, i \leqslant n$, are nonnegative and $\eta$ has arbitrary $\alpha$-stable distribution. Thus, in the case $\alpha=2 Z_{n}$ for all $n$ is a standard normal random variable. In the general case, the distribution of $Z_{n}$ depends on $n$, and $Z_{n}$ (as well as $S_{n}$ ) does not need to converge to a limit distribution. However, approximation of $S_{n}$ by $Z_{n}$ is very natural and the present note discusses the rate of such approximation, namely, the rate of convergence as $n \rightarrow \infty$ of the quantity $\Delta_{n}$ from (5) with $S_{n}$ and $Z_{n}$ from (8). Introduce the «Lyapunov fraction»

$$
L_{n}(\alpha, \delta):=\left(\frac{B_{n}(\alpha+\delta)}{B_{n}(\alpha)}\right)^{\alpha+\delta}=\frac{\sum_{i \leqslant n}\left|b_{n, i}\right|^{\alpha+\delta}}{\left(\sum_{i \leqslant n}\left|b_{n, i}\right|^{\alpha}\right)^{(\alpha+\delta) / \alpha}} .
$$

Theorem 1. Let $\left(\varepsilon_{i}\right)$ be i.i.d. whose distribution satisfies Assumption $\mathrm{D}(\alpha, \delta)$, for some $0<\alpha \leqslant 2, \alpha \neq 1,0<\delta \leqslant 1$. Then

$$
\Delta_{n} \leqslant K \begin{cases}\mu_{2+\delta} L_{n}(2, \delta), & \text { if } \alpha=2, \\ \max \left\{\kappa_{\alpha, \delta},\left(\kappa_{\alpha, \delta}\right)^{1 /(\alpha+\delta+1)}\right\} L_{n}(\alpha, \delta), & \text { if } 0<\alpha<2, \alpha \neq 1,\end{cases}
$$

where $K$ is a constant depending only on $\alpha, \delta$.

$\mathrm{R} \mathrm{e} \mathrm{m} \mathrm{a} \mathrm{r} \mathrm{k} \mathrm{1.} \mathrm{For} \alpha=2$, Theorem 1 follows from the classical estimate in $[15$, Theorem 5.7], and for $0<\alpha<2$, from [14, Corollary 1] (see also [4] and [13]). Although the above mentioned results refer to finite sums of independent r.v.'s only, they can easily be extended to the situation in Theorem 1, by truncating infinite sums in (8) and then letting the level of truncation grow to infinity.

$\mathrm{R}$ e $\mathrm{m}$ a $\mathrm{rk} 2$. The reason for separating the estimates in the cases $\alpha=2$ (Gaussian approximation) and $0<\alpha<2$ (stable approximation) is that, traditionally, in the former case one uses moments, while in the latter case moments of the order exceeding $\alpha$ do not exist and one has to use pseudomoments. It is possible to use pseudomoments in the Gaussian case too and to replace the two lines in (11) by a single estimate which holds for any $0<\alpha \leqslant 2, \alpha \neq 1$. To do this, instead of the estimate in [15], one has to use a result due to Nagaev and Rotar' [12] giving a rate of normal approximation in terms of (finite) pseudomoment $\kappa_{2,1}$. The discussion in [13] and [14] shows that extension of these results to the case of finite pseudomoment $\kappa_{2, \delta}$ is not difficult.

3. Rate of stable approximation for sums of moving averages. In this section Theorem 1 is applied to estimate the quantity $\Delta_{n}$ in (5). We compare sums $S_{n}, Z_{n}$ of moving averages $X_{t}, Y_{t}$ in (1) and (4), respectively, which can be rewritten as the corresponding weighted sums in (8), with weights $b_{n, i}$ given by

$$
b_{n, i}=\sum_{t=1 \vee i}^{n} a_{t-i} \quad(i \leqslant n) .
$$

If $\left(\varepsilon_{i}\right)$ satisfy Assumption $\mathrm{D}(\alpha, \delta)$, the series in (1), (4) converge a.s. provided the moving average coefficients satisfy

$$
\sum_{j=0}^{\infty}\left|a_{j}\right|^{\alpha}<\infty
$$

Theorem 1 reduces estimation of the uniform distance $\Delta_{n}$ between probability distributions of $S_{n}$ and the $\alpha$-stable r.v. $Z_{n}$ in (8) to that of $L_{n}(\alpha, \delta)$ which depends only on weights $\left(b_{n, i}\right)$ in (12), or on the moving average coefficients $\left(a_{j}\right)$ in (1). In this section we study the behavior of $L_{n}(\alpha, \delta)$ under various assumptions on $\left(a_{j}\right)$ and apply the results to estimation of $\Delta_{n}$.

Introduce the following notation. Given two sequences $\left(a_{n}, n \geqslant 0\right)$ and $\left(b_{n}, n \geqslant 0\right)$, we write $a_{n} \simeq b_{n}$ whenever the inequality $C_{1} b_{n} \leqslant a_{n} \leqslant C_{2} b_{n}$ holds for all $n \geqslant 0$ and some constants $0<C_{1}<C_{2}<\infty$, and $a_{n} \sim b_{n}$ whenever $\lim _{n \rightarrow \infty} a_{n} / b_{n}=1$. Recall that a sequence $\left(b_{j}, j \geqslant 1\right)$ is regularly varying with index $\gamma \in \mathbf{R}$ if $b_{j}$ can be represented as $b_{j}=\Lambda(j) j^{\gamma}$, where $\Lambda(x), x \in[1, \infty)$, is slowly varying at infinity and bounded on 
bounded intervals. Let $\left(b_{j}\right)$ be regularly varying with index $\gamma$, and $a_{j} \sim b_{j}$, then $\left(a_{j}\right)$ is also regularly varying with index $\gamma$. On the other hand, if $\left(b_{j}\right)$ is regularly varying with index $\gamma$ and $a_{j} \simeq b_{j}$, then $\left(a_{j}\right)$ need not be regularly varying. (Take, e.g., $b_{j}=(1+j)^{\gamma}$ and $a_{j}=b_{j}\left(2+(-1)^{j}\right)$.)

Consider first the case of positive coefficients $\left(a_{j}\right)$ which are not necessarily regularly varying but are bounded from both sides by positive regularly varying sequences. Note that in this case $\mathbf{P}\left\{Z_{n} \leqslant x\right\}=\mathbf{P}\{\eta \leqslant x\}$ does not depend on $n$ and coincides with the distribution of $\eta$ in (7).

Theorem 2. Let $S_{n}=B_{n}^{-1} \sum_{t=1}^{n} X_{t}$, where $X_{t}$ is a moving average process in (1), with i.i.d. innovations $\left(\varepsilon_{j}\right)$ satisfying Assumption $\mathrm{D}(\alpha, \delta), 0<\alpha<2, \alpha \neq 1,0<\delta \leqslant 1$. Let $a_{j} \simeq(1+j)^{-\beta}$, for some $\beta>\alpha^{-1}$. Then

$$
\Delta_{n} \leqslant C_{1} n^{-\delta / \alpha}
$$

where $C_{1}$ is some constant depending on $\alpha, \beta, \delta$ as well as on $\mathbf{E}|\varepsilon|^{2+\delta} \quad(\alpha=2)$ or the pseudomoment $\kappa_{\alpha, \delta}(0<\alpha<2)$ only. Moreover,

$$
B_{n}(\alpha) \simeq \begin{cases}n^{1-\beta+(1 / \alpha)}, & \text { if } \alpha^{-1}<\beta<1, \alpha>1, \\ n^{1 / \alpha}, & \text { if } \beta>1, \\ n^{1 / \alpha} \ln n, & \text { if } \beta=1, \alpha>1 .\end{cases}
$$

$\mathrm{P}$ r o o f. According to Theorem 1, the definitions of $L_{n}(\alpha, \delta), B_{n}(\alpha), b_{n, i}$ (see (10), (9), (12)), and assumption $a_{j} \simeq(1+j)^{-\beta}$, it suffices to consider the case $a_{j}=(1+j)^{-\beta}$ only. Then (13) follows from (11) and (14), while (14) for $a_{j}=(1+j)^{-\beta}$ follows by direct calculation (see also the proof of Theorem 3 below). Theorem 2 is proved.

Next, we discuss the case when the moving average coefficients $\left(a_{j}\right)$ are regularly varying or absolutely summable and may change their sign. The following classification in terms of memory is often used to characterize the dependence structure and the limit behavior of various functionals of $X_{t}$ (see, e.g., [6]). Correspondingly, we shall distinguish between the following cases, or assumptions:

(I) $\sum_{j=0}^{\infty}\left|a_{j}\right|<\infty, \sum_{j=0}^{\infty} a_{j} \neq 0$;

(II) $a_{j}=\Lambda(j) j^{-\beta}, \alpha^{-1}<\beta<1,1<\alpha \leqslant 2, \Lambda$ is slowly varying at infinity;

(III) $a_{j}=\Lambda(j) j^{-\beta}, \sum_{j=0}^{\infty} a_{j}=0, \beta>1, \Lambda$ is slowly varying at infinity.

Cases (I), (II), (III) are usually called short memory, long memory and negative memory, respectively. The following theorem shows that in cases (I) and (II) the rate of $\alpha$-stable approximation is the same as in the i.i.d. case.

Theorem 3. Let $X_{t}$ be a moving average as in $(1)$, with i.i.d. innovations $\left(\varepsilon_{j}\right)$ satisfying Assumption $\mathrm{D}(\alpha, \delta), 0<\alpha \leqslant 2, \alpha \neq 1,0<\delta \leqslant 1$. Assume that the moving average coefficients $\left(a_{j}\right)$ satisfy one of assumptions (I)-(III) above. Then:

(i) Under assumptions (I) or (II) (short or long memory),

$$
\Delta_{n} \leqslant C_{2} n^{-\delta / \alpha}
$$

(ii) under assumption (III) (negative memory)

$$
\Delta_{n} \leqslant C_{2}\left\{\begin{array}{r}
n^{-\delta / 2}, \quad \text { if } 1 \vee \alpha^{-1}<\beta<1+(\alpha+\delta)^{-1}, \\
\left(\Lambda(n) n^{1+1 / \alpha-\beta}\right)^{-(\alpha+\delta)}=O\left(B_{n}(\alpha)^{-(\alpha+\delta)}\right), \\
\text { if } 1+(\alpha+\delta)^{-1}<\beta<1+\alpha^{-1} .
\end{array}\right.
$$

In (15) and (16), $C_{2}$ is a constant depending on the sequence $\left(a_{j}\right)$ as well as on the $(2+\delta)$-moment $(\alpha=2)$ or the pseudomoment $\kappa_{\alpha, \delta}(0<\alpha<2)$ only, otherwise independent of the distribution of $\varepsilon$.

Theorem 3 follows easily from Theorem 1 and Lemma 1 below, which gives asymptotical behavior of $B_{n}(\alpha)$ under conditions (I)-(III).

$\mathrm{R}$ e $\mathrm{m}$ a $\mathrm{rk} 3$. It is not difficult to explicitly give the dependence of constants $C_{1}, C_{2}$ in Theorems 2 and 3 on moments and pseudomoments. Namely, similarly to (11), one can show that there are some constants $\widetilde{C}_{i}, i=1,2$, depending on $\alpha, \delta$, and the sequence $\left(a_{j}\right)$ only, such that $C_{i}=\mu_{2+\delta} \widetilde{C}_{i}$ if $\alpha=2$, and $C_{i}=\max \left\{\kappa_{\alpha, \delta},\left(\kappa_{\alpha, \delta}\right)^{1 /(\alpha+\delta+1)}\right\} \widetilde{C}_{i}$ if $0<\alpha<2$. 
$\mathrm{R}$ e $\mathrm{m}$ a r k 4. An open question is whether the rate of convergence in case (III), $1 \vee \alpha^{-1}<\beta<1+(\alpha+\delta)^{-1}$, given in (16), is optimal. The fact that $B_{n}(\alpha)$ is bounded for $\beta>1+(\alpha+\delta)^{-1}$ is in favor of a positive answer to this question.

In Lemma 1 below $\Lambda(x), x \geqslant 0$, is a function slowly varying at infinity, which we assume to be strictly positive for $x$ large enough.

Lemma 1. (i) Let $\sum_{j=0}^{\infty}\left|a_{j}\right|^{1 \wedge \alpha}<\infty, \sum_{j=0}^{\infty} a_{j} \neq 0, \alpha>0$. Then

$$
B_{n}^{\alpha}(\alpha) \sim\left|\phi_{\infty}\right|^{\alpha} n
$$

where $\phi_{\infty}:=\sum_{i=0}^{\infty} a_{i}$.

(ii) Let $a_{j}=\Lambda(j) j^{-\beta}$, where $\alpha^{-1}<\beta<1, \alpha>1$. Then

$$
B_{n}^{\alpha}(\alpha) \sim c(\alpha, \beta) \Lambda^{\alpha}(n) n^{\alpha(1-\beta)+1}
$$

where the constant $c(\alpha, \beta)$ is given in (19) below.

(iii) Let $a_{j}=\Lambda(j) j^{-\beta}$, where $1 \vee \alpha^{-1}<\beta<1+\alpha^{-1}, \alpha>0 ; \sum_{j=0}^{\infty} a_{j}=0$. Then relation (18) holds, with $c(\alpha, \beta)$ given in (19).

(iv) Let $a_{j}=\Lambda(j) j^{-\beta}$, where $\beta>1+\alpha^{-1}, \alpha>0 ; \sum_{j=0}^{\infty} a_{j}=0$. Then $B_{n}^{\alpha}(\alpha) \rightarrow$ $2 \sum_{i=0}^{\infty}\left|\sum_{j=i+1}^{\infty} a_{j}\right|^{\alpha}>0$.

P r o o f. (i) Note that $\sum_{j=0}^{\infty}\left|a_{j}\right|^{1 \wedge \alpha}<\infty$ implies $\sum_{j=0}^{\infty}\left|a_{j}\right|<\infty$, for any $\alpha>0$. Let $\phi_{n}:=\sum_{i=0}^{n} a_{i}, \Phi_{n}:=\sum_{i \geqslant n}\left|a_{i}\right|, n \geqslant 0$. Then $\left|\phi_{n+i}-\phi_{i}\right| \leqslant \Phi_{i+1}(i, n \geqslant 0), \phi_{n} \rightarrow \phi_{\infty}$, $\Phi_{n} \rightarrow 0(n \rightarrow \infty)$. Write

$$
n^{-1} B_{n}^{\alpha}(\alpha)=D_{n}^{\prime}+D_{n}^{\prime \prime}, \quad D_{n}^{\prime}:=n^{-1} \sum_{i=0}^{n-1}\left|\phi_{i}\right|^{\alpha}, \quad D_{n}^{\prime \prime}:=n^{-1} \sum_{i=0}^{\infty}\left|\phi_{n+i}-\phi_{i}\right|^{\alpha} .
$$

By the dominated convergence theorem, $\lim _{n \rightarrow \infty} D_{n}^{\prime}=\left|\phi_{\infty}\right|^{\alpha}$. It remains to show that $D_{n}^{\prime \prime}=o(1)$. Assume first $\alpha \geqslant 1$. Then $\left|\phi_{n+i}-\phi_{i}\right|^{\alpha} \leqslant \Phi_{0}^{\alpha-1}\left|\phi_{n+i}-\phi_{i}\right|$ and therefore $D_{n}^{\prime \prime} \leqslant \Phi_{0}^{\alpha-1} n^{-1} \sum_{i=0}^{\infty}\left(\left|a_{i+1}\right|+\cdots+\left|a_{n+i}\right|\right) \leqslant \Phi_{0}^{\alpha-1} n^{-1}\left(\Phi_{0}+\cdots+\Phi_{n}\right) \longrightarrow 0 \quad(n \rightarrow \infty)$.

Next, let $0<\alpha<1$. Then $\sum_{j=0}^{\infty}\left|a_{j}\right|^{\alpha}<\infty$ and we similarly obtain

$$
D_{n}^{\prime \prime} \leqslant n^{-1} \sum_{i=0}^{\infty}\left(\left|a_{i+1}\right|^{\alpha}+\cdots+\left|a_{n+i}\right|^{\alpha}\right) \leqslant n^{-1} \sum_{i=1}^{n} \sum_{j=i}^{\infty}\left|a_{j}\right|^{\alpha} \rightarrow 0 \quad(n \rightarrow \infty),
$$

proving (17).

(ii) Let $\phi_{n}=\sum_{i=0}^{n} a_{i}$ as in part (i). Observe that, by the dominated convergence theorem,

$$
\gamma_{n}:=\frac{c_{n}}{\Lambda(n) n^{1-\beta}}=\sum_{i=0}^{n} \frac{\Lambda(i)}{\Lambda(n)}\left(\frac{i}{n}\right)^{-\beta} \frac{1}{n} \longrightarrow \int_{0}^{1} x^{-\beta} \mathrm{d} x=(1-\beta)^{-1} .
$$

Then $B_{n}^{\alpha}(\alpha) /\left(\Lambda^{\alpha}(n) n^{\alpha(1-\beta)+1}\right)=D_{n}+D_{n}^{\prime}(K)+D_{n}^{\prime \prime}(K)$, where

$$
\begin{aligned}
D_{n} & :=\sum_{i=0}^{n}\left|\gamma_{i}\right|^{\alpha} \frac{\Lambda^{\alpha}(i)}{\Lambda^{\alpha}(n)}\left(\frac{i}{n}\right)^{\alpha(1-\beta)} \frac{1}{n} \\
D_{n}^{\prime}(K) & :=\sum_{i=0}^{K n}\left|\gamma_{n+i} \frac{\Lambda(n+i)}{\Lambda(n)}\left(\frac{n+i}{n}\right)^{1-\beta}-\gamma_{i} \frac{\Lambda(i)}{\Lambda(n)}\left(\frac{i}{n}\right)^{1-\beta}\right|^{\alpha} \frac{1}{n} \\
D_{n}^{\prime \prime}(K) & :=\Lambda^{-\alpha}(n) n^{-\alpha(1-\beta)-1} \sum_{i>K n}\left|\phi_{n+i}-\phi_{i}\right|^{\alpha},
\end{aligned}
$$

where $K$ is a large number. By the dominated convergence theorem, for any $K<\infty$,

$$
\begin{aligned}
D_{n} & \longrightarrow(1-\beta)^{-\alpha} \int_{0}^{1} x^{\alpha(1-\beta)} \mathrm{d} x=: D \\
D_{n}^{\prime}(K) & \longrightarrow(1-\beta)^{-\alpha} \int_{0}^{K}\left((1+x)^{1-\beta}-x^{1-\beta}\right)^{\alpha} \mathrm{d} x=: D^{\prime}(K) .
\end{aligned}
$$


Rewrite

$$
D_{n}^{\prime \prime}(K)=n^{-\alpha} \sum_{i>n K}\left|\sum_{j=1}^{n} \frac{\Lambda(i+j)}{\Lambda(n)}\left(\frac{i+j}{n}\right)^{-\beta}\right|^{\alpha} \frac{1}{n} .
$$

We shall use the well-known property of slowly varying functions: for any $\theta>0$,

$$
\sup _{x \in[1, \infty)} \frac{1}{x^{\theta}}\left|\frac{\Lambda(n x)}{\Lambda(n)}-1\right| \rightarrow 0 \quad(n \rightarrow \infty) .
$$

Therefore, for any (sufficiently small) $\theta>0$ one can find a constant $C=C_{\theta}$ such that

$$
\begin{aligned}
D_{n}^{\prime \prime}(K) & \leqslant C n^{-\alpha} \sum_{i>n K}\left(\sum_{j=1}^{n}\left(\frac{i+j}{n}\right)^{\theta-\beta}\right)^{\alpha} \frac{1}{n} \leqslant C \int_{K}^{\infty}\left(\int_{0}^{1}(x+y)^{\theta-\beta} \mathrm{d} y\right)^{\alpha} \mathrm{d} x \\
& \leqslant C \int_{K}^{\infty} x^{\alpha(\theta-\beta)} \mathrm{d} x \leqslant C K^{1+\alpha(\theta-\beta)}=o(1) \quad(K \rightarrow \infty),
\end{aligned}
$$

provided $\theta>0$ was chosen so that $\theta<\beta-\alpha^{-1}$. Furthermore, $D^{\prime}(K) \rightarrow$ $D^{\prime}:=(1-\beta)^{-\alpha} \int_{0}^{\infty}\left((1+x)^{1-\beta}-x^{1-\beta}\right)^{\alpha} \mathrm{d} x$ as $K \rightarrow \infty$. We have proved that $\lim _{n \rightarrow \infty} B_{n}^{\alpha}(\alpha) /\left(\Lambda^{\alpha}(n) n^{\alpha(1-\beta)+1}\right)=D+D^{\prime}=: c(\alpha, \beta)$, where

$$
c(\alpha, \beta)=|1-\beta|^{-\alpha}\left(\int_{0}^{1} x^{\alpha(1-\beta)} \mathrm{d} x+\int_{0}^{\infty}\left|(1+x)^{1-\beta}-x^{1-\beta}\right|^{\alpha} \mathrm{d} x\right) .
$$

In particular,

$$
c(2, \beta)=\frac{\Gamma^{2}(2-\beta)}{(1-\beta)^{2} \Gamma(4-2 \beta)|\cos (\pi \beta)|},
$$

see $[17,(9.3)]$.

(iii) Observe that $\phi_{n}=-\sum_{i=n+1}^{\infty} a_{i}$ and

$$
\gamma_{n}:=\frac{\phi_{n}}{\Lambda(n) n^{1-\beta}}=-\sum_{i=n+1}^{\infty} \frac{\Lambda(i)}{\Lambda(n)}\left(\frac{i}{n}\right)^{-\beta} \frac{1}{n} \longrightarrow-\int_{1}^{\infty} x^{-\beta} \mathrm{d} x=-\frac{1}{\beta-1} .
$$

The rest of the proof of (18) is completely analogous to the case (ii).

(iv) Follows by $\sum_{i=0}^{\infty}\left|\sum_{j=i+1}^{\infty} a_{j}\right|^{\alpha}<\infty$. Lemma 1 is proved.

We end the paper with two examples where the classical approximation rate $n^{-\delta / \alpha}$ for $S_{n}=B_{n}^{-1} \sum_{t=1}^{n} X_{t}$ by $\alpha$-stable $Z_{n}=B_{n}^{-1} \sum_{t=1}^{n} Y_{t}$ takes place. In the first example, the distribution of $Z_{n}$ does not depend on $n$ and coincides with the limit distribution for $S_{n}$ but the normalizing sequence $B_{n}$ is not regularly varying, so that partial sums process of $X_{t}$ does not converges (see Introduction). In the second example, the approximating $\alpha$-stable distribution $Z_{n}$ does not converge as its skewness parameter oscillates with $n$.

$\mathrm{E} \times \mathrm{a} \mathrm{m} \mathrm{p} \mathrm{l} \mathrm{e} 1$. Let $n(k) \geqslant 1(k=1,2, \ldots)$ be an increasing sequence of integers such that $\lim _{k \rightarrow \infty} n(k+1) / n(k)=\infty$.

Fix $\beta \in\left(\alpha^{-1}, 1\right), 1<\alpha \leqslant 2$. Set

$$
a_{j}=(1+j)^{-\beta} \begin{cases}2, & \text { if } \quad n(k) \leqslant j<n(k+1), \quad k \text { odd } \\ 1, & \text { if } n(k) \leqslant j<n(k+1), \quad k \text { even. }\end{cases}
$$

Clearly, $a_{j} \simeq(1+j)^{-\beta}$ and therefore $B_{n}=B_{n}(\alpha) \simeq n^{1-\beta+\alpha^{-1}}$, see Theorem 2. To show that $\left(B_{n}\right)$ is not regulary varying, it suffices to show that $\left(B_{n}^{\alpha}\right)$ is not regularily varying with index $H:=\alpha(1-\beta)+1$. The last fact follows from

$$
\liminf _{k \rightarrow \infty} \frac{B_{2 n(2 k)}^{\alpha}}{B_{n(2 k)}^{\alpha}}<2^{H} .
$$

Similarly as in the proof of Lemma 1 (ii),

$$
B_{n}^{\alpha} \sim \int_{0}^{n}\left(\int_{0}^{x} u^{-\beta} h(u) \mathrm{d} u\right)^{\alpha} \mathrm{d} x+\int_{0}^{\infty}\left(\int_{x}^{n+x} u^{-\beta} h(u) \mathrm{d} u\right)^{\alpha} \mathrm{d} x=: D_{n}^{\prime}+D_{n}^{\prime \prime},
$$


where $h(u):=2$ if $n(k) \leqslant u<n(k+1)$ and $k$ is odd, $h(u):=1$ if $n(k) \leqslant u<n(k+1)$ and $k$ is even. Note $n(2 k)$ is even and therefore

$$
h(u)= \begin{cases}2, & n(2 k-1) \leqslant u<n(2 k), \\ 1, & n(2 k) \leqslant u<n(2 k+1) .\end{cases}
$$

Using $n(2 k-1) / n(2 k) \rightarrow 0, n(2 k+1) / n(2 k) \rightarrow \infty$ and definition of $h(u)$, it is easy to show that

$$
\begin{aligned}
& D_{n(2 k)}^{\prime} \sim 2^{\alpha} \int_{0}^{n(2 k)}\left(\int_{0}^{x} u^{-\beta} \mathrm{d} u\right)^{\alpha} \mathrm{d} x=2^{\alpha}(n(2 k))^{H} c_{1}, \\
& D_{n(2 k)}^{\prime \prime} \sim \int_{0}^{\infty}\left(\int_{x}^{n(2 k)+x} u^{-\beta} \mathrm{d} u\right)^{\alpha} \mathrm{d} x=(n(2 k))^{H} c_{2},
\end{aligned}
$$

where

$$
c_{1}:=\int_{0}^{1}\left(\int_{0}^{x} u^{-\beta} \mathrm{d} u\right)^{\alpha} \mathrm{d} x, \quad c_{2}:=\int_{0}^{\infty}\left(\int_{x}^{1+x} u^{-\beta} \mathrm{d} u\right)^{\alpha} \mathrm{d} x .
$$

In a similar way,

$$
D_{2 n(2 k)}^{\prime \prime} \sim \int_{0}^{\infty}\left(\int_{x}^{2 n(2 k)+x} u^{-\beta} \mathrm{d} u\right)^{2} \mathrm{~d} x=2^{\alpha(1-\beta)+1}(n(2 k))^{H} c_{2},
$$

while

$$
\begin{aligned}
D_{2 n(2 k)}^{\prime} & \sim D_{n(2 k)}^{\prime}+\int_{n(2 k)}^{2 n(2 k)}\left(\int_{0}^{x} u^{-\beta} h(u) \mathrm{d} u\right)^{\alpha} \mathrm{d} x \\
& \sim D_{n(2 k)}^{\prime}+\int_{n(2 k)}^{2 n(2 k)}\left(2 \int_{0}^{n(2 k)} u^{-\beta} \mathrm{d} u+\int_{n(2 k)}^{x} u^{-\beta} \mathrm{d} u\right)^{\alpha} \mathrm{d} x \\
& =D_{n(2 k)}^{\prime}+(n(2 k))^{H} c_{3},
\end{aligned}
$$

where $c_{3}:=(1-\beta)^{-\alpha} \int_{1}^{2}\left(1+x^{1-\beta}\right)^{\alpha} \mathrm{d} x$. From (21)-(24) we obtain

$$
\frac{B_{2 n(2 k)}^{\alpha}}{B_{n(2 k)}^{\alpha}} \sim \frac{D_{2 n(2 k)}^{\prime}+D_{2 n(2 k)}^{\prime \prime}}{D_{n(2 k)}^{\prime}+D_{n(2 k)}^{\prime \prime}} \sim \frac{2^{\alpha} c_{1}+c_{3}+2^{\alpha(1-\beta)+1} c_{2}}{2^{\alpha} c_{1}+c_{2}} .
$$

The desired inequality (20) now follows from $c_{3}<2^{\alpha} c_{1}\left(2^{\alpha(1-\beta)+1}-1\right)$, or

$$
\int_{1}^{2}\left(1+x^{1-\beta}\right)^{\alpha} \mathrm{d} x<\frac{2^{\alpha}\left(2^{\alpha(1-\beta)+1}-1\right)}{1+\alpha(1-\beta)} .
$$

The last inequality holds because the right-hand side equals $\int_{1}^{2}\left(2 x^{1-\beta}\right)^{\alpha} \mathrm{d} x$. This proves (20).

$\mathrm{E} \times \mathrm{a} \mathrm{mp} 1 \mathrm{e} 2$. Let $n(k) \geqslant 1, n(k+1) / n(k) \rightarrow \infty$, be the same as in Example 1. For $\beta \in\left(\alpha^{-1}, 1\right), 1<\alpha<2$, let

$$
a_{j}=(-1)^{k}(1+j)^{-\beta}, \quad n(k) \leqslant j<n(k+1), \quad k \geqslant 1 .
$$

The above sequence $\left(a_{j}\right)$ is not regularly varying and does not satisfy the conditions of Theorem 2 nor Theorem 3. However, Theorem 1 is applicable, since

$$
B_{n}^{\alpha}=\sum_{i \leqslant n}\left|b_{n, i}\right|^{\alpha} \simeq n^{\alpha(1-\beta)+1},
$$

see below, and therefore $\Delta_{n}=\sup _{x}\left|\mathbf{P}\left\{S_{n} \leqslant x\right\}-\mathbf{P}\left\{Z_{n} \leqslant x\right\}\right| \leqslant C n^{-\delta / \alpha}$, provided the i.i.d. r.v.'s $\left(\varepsilon_{i}\right)$ satisfy assumption $\mathrm{D}(\alpha, \delta)$. Let $c_{\varepsilon}^{+} \neq c_{\varepsilon}^{-}$in (6) so that $\eta$ in (7) is not symmetric and therefore $Z_{n}=\sum_{i \leqslant n} b_{n, i} \eta_{i}$ is not symmetric. 
The skewness parameter of $Z_{n}$ equals $\beta_{\eta} Q_{n}$, where $\beta_{\eta}:=\left(c_{\varepsilon}^{+}-c_{\varepsilon}^{-}\right) /\left(c_{\varepsilon}^{+}+c_{\varepsilon}^{-}\right)$is the skewness parameter of $\eta$ in (7) and

$$
Q_{n}=B_{n}^{-\alpha} \sum_{i \leqslant n}\left|b_{n, i}\right|^{\alpha} \operatorname{sign}\left(b_{n, i}\right)
$$

Clearly, if $\beta_{\eta} \neq 0$ and the sequence $\left(Q_{n}\right)$ does not converge, i.e.,

$$
\limsup Q_{n}>\liminf Q_{n}
$$

then $\left(Z_{n}\right)$ does not converge, too (in distribution). Let us prove (25). The relation $B_{n}^{\alpha}=O\left(n^{\alpha(1-\beta)+1}\right)$ follows immediately from $\left|a_{j}\right| \leqslant(1+j)^{-\beta}$, therefore it suffices to show the lower bound

$$
B_{n}^{\alpha}>c_{1} n^{\alpha(1-\beta)+1}
$$

for some constant $c_{1}>0$. Let $\phi_{n}:=\sum_{i=0}^{n} a_{i}$. We claim that there are constants $c_{2}, K>0$ such that for any $k$

$$
\left|\phi_{i}\right|>c_{2} i^{1-\beta}, \quad \operatorname{sign}\left(\phi_{i}\right)=(-1)^{k} \quad(K n(k)<i \leqslant n(k+1)) .
$$

For $n(k)<n \leqslant n(k+1)$, by definition of $a_{j}$, we have

$$
\phi_{n}-\phi_{n(k)}=\sum_{j=n(k)+1}^{n} a_{j}=(-1)^{k} \sum_{j=n(k)+1}^{n}(1+j)^{-\beta} .
$$

Hence, there is a constant $c_{5}>0$ such that

$$
\left|\phi_{i}-\phi_{n(k)}\right|>c_{5} i^{1-\beta}, \quad \operatorname{sign}\left(\phi_{i}-\phi_{n(k)}\right)=(-1)^{k}
$$

holds for all $2 n(k)<i \leqslant n(k+1)$. Take $c_{2}=c_{5} / 2$, then

$\left|\phi_{i}\right| \geqslant\left|\phi_{i}-\phi_{n(k)}\right|-\left|\phi_{n(k)}\right|>c_{5} i^{1-\beta}-(1-\beta)^{-1}(n(k))^{1-\beta}>c_{2} i^{1-\beta}, \quad \operatorname{sign}\left(\phi_{i}\right)=(-1)^{k}$, holds for all $K n(k)<i \leqslant n(k+1)$, provided $K$ is chosen large enough. This proves (28).

Now (27) follows from (28). Indeed, if $n>2 K n(k)$, then

$$
B_{n}^{\alpha} \geqslant \sum_{K n(k)<i \leqslant n}\left|\phi_{i}\right|^{\alpha}>c_{2}^{\alpha} \sum_{K n(k)<i \leqslant n} i^{\alpha(1-\beta)}>c_{3} n^{\alpha(1-\beta)+1},
$$

for some constant $c_{3}>0$ independent of $n$. Similarly, if $n \leqslant 2 K n(k)$, or $n(k) \geqslant n /(2 K)$, then

$$
B_{n}^{\alpha}>c_{1}^{\alpha} \sum_{2 K n(k-1)<i \leqslant n(k)} i^{\alpha(1-\beta)}>c_{4}(n(k))^{\alpha(1-\beta)+1}>c_{4} n^{\alpha(1-\beta)+1}
$$

for some constant $c_{4}>0$ independent of $n$. Thus, we proved (27) and (25).

Let us check (26). It suffices to take the limsup and liminf along a subsequence $\bar{n}(k) \rightarrow \infty, \bar{n}(k):=(n(k)+n(k+1)) / 2$. The integer $\bar{n}(k)$ is in middle of the interval $(n(k), n(k+1))$ and $\bar{n}(k) / n(k) \rightarrow \infty$ according to our construction of $(n(k))$. We have $Q_{n}=Q_{n, 1}+Q_{n, 2}$, where $Q_{n, 1}:=B_{n}^{-\alpha} \sum_{i=0}^{n}\left|b_{n, i}\right|^{\alpha} \operatorname{sign}\left(b_{n, i}\right), \quad Q_{n, 2}:=$ $B_{n}^{-\alpha} \sum_{i<0}\left|b_{n, i}\right|^{\alpha} \operatorname{sign}\left(b_{n, i}\right)$. Note that $Q_{n, 1}=B_{n}^{-\alpha} \sum_{i=0}^{n}\left|\phi_{i}\right|^{\alpha} \operatorname{sign}\left(\phi_{i}\right)$. Using (28),

$$
Q_{n, 1}=B_{n}^{-\alpha} \sum_{i=0}^{K n(k)}\left|\phi_{i}\right|^{\alpha} \operatorname{sign}\left(\phi_{i}\right)+(-1)^{k} B_{n}^{-\alpha} \sum_{i=K n(k)+1}^{n}\left|\phi_{i}\right|^{\alpha}=: Q_{n, 3}+(-1)^{k} Q_{n, 4} .
$$

Then (26) follows from

$$
\liminf _{k \rightarrow \infty} Q_{\bar{n}(k), 4}>0, \quad \limsup _{k \rightarrow \infty}\left|Q_{\bar{n}(k), i}\right|=0 \quad(i=2,3) .
$$

The proof of (29) follows from $B_{n}^{\alpha} \simeq n^{\alpha(1-\beta)+1}$, see (25), and a similar argument as in Example 1. 


\section{REFERENCES}

1. Аркашов Н.С., Борисов И.С. Гауссовская аппроксимация процессов частных сумм скользяших средних. - Сиб. матем. журн., 2004, т. 45, № 6, с. 1221-1255.

2. Астраускас $A$. Предельные теоремы для сумм линейно порожденных случайных величин. - Литов. матем. сб., 1983, т. 23, № 2, с. 3-12.

3. Avram F., Taqqu M.S. Weak convergence of sums of moving averages in the $\alpha$-stable domain of attraction. - Ann. Probab., 1992, v. 20, № 1, p. 483-503.

4. Christoph G., Wolf W. Convergence Theorems with a Stable Limit Law. Berlin: Akademie-Verlag, 1992, 200 p. (Math. Res., v. 70.)

5. Давыдов Ю.А. Принцип инвариантности для стационарных процессов. - Теория вероятн. и ее примен., 1970 , т. 15 , в. 3 , с. $498-509$.

6. Giraitis L., Surgailis D. ARCH-type bilinear models with double long memory. Stochastic Process. Appl., 2002, v. 100, p. 275-300.

7. Городецкий В.В. О сходимости к полуустойчивым гауссовским процессам. - Теория вероятн. и ее примен., 1977 , т. 22 , в. 3 , с. 513-522; исправл.: 1979 , т. 24 , B. 2.

8. Ибрагимов И. А. Некоторые предельные теоремы для стационарных в узком смысле вероятностных процессов. - Докл. АН СССР, 1959, т. 125, № 4, с. 711-714.

9. Ибрагимов И. А., Линник Ю. В. Независимые и стационарно связанные величины. М.: Наука, 1965, 524 с.

10. Lamperti J. W. Semi-stable stochastic processes. - Trans. Amer. Math. Soc., 1962, v. 104 , p. $62-78$.

11. Maejima $M$. On a class of self-similar processes. - Z . Wahrscheinlichkeitstheor. Verw. Geb., 1983, v. 62, № 2, p. 235-245.

12. Нагаев С. В., Ротарь В. И. Об усилении оценок типа Ляпунова (случай близости распределений слагаемых к нормальному). - Теория вероятн. и ее примен., 1973, т. 18 , в. 1 , с. $109-121$; исправл.: 1976 , т. 21 , в. 1.

13. Паулаускас В. И. Оценки остаточного члена в предельной теореме в случае устойчивого предельного закона. - Литов. матем. сб., 1974, т. 14, № 1, с. 165-187.

14. Паулаускас В. И. Равномерные и неравномерные оценки остаточного члена в предельной теореме с устойчивым предельным законом. - Литов. матем. сб., 1974, т. 14, № 4, c. 171-185.

15. Petrov $V . V$. Limit Theorems of Probability Theory. Sequences of Independent Random Variables. New York: Clarendon Press, 1995, 292 p. (Oxford. Stud. Probab., v. 4.)

16. Сургайлис Д. О сходимости сумм нелинейных функций от скользящих средних к автомодельным процессам. - Докл. АН СССР, 1981, т. 257, № 1, с. 51-54.

17. Taqqu M. S. Fractional Brownian motion and long-range dependence. - Theory and Applications of Long-Range Dependence. Ed. by P. Doukhan, G. Oppenheim, and M.S. Taqqu. Boston: Birkhäuser, 2003, p. 5-38.

Поступила в редакцию 4.V.2006

Из-за технической ошибки на первых страницах выпусков журнала, начиная с тома 49, выпуск 2, 2004 года, в строке 6 сверху следует читать «Москва. Наука». 